\title{
Intraoperative near-infrared imaging with receptor-specific versus passive delivery of fluorescent agents in pituitary adenomas
}

\author{
Steve S. Cho, BS, ${ }^{1,4}$ Jun Jeon, BS, ${ }^{1,4}$ Love Buch, BS, ${ }^{1}$ Shayoni Nag, BA, ${ }^{1}$ \\ MacLean Nasrallah, MD, PhD, ${ }^{2}$ Philip S. Low, PhD, ${ }^{5}$ M. Sean Grady, MD, ${ }^{1}$ Sunil Singhal, MD, ${ }^{3}$ and \\ John Y. K. Lee, MD, MSCE'
}

Departments of ${ }^{1}$ Neurosurgery, ${ }^{2}$ Pathology, and ${ }^{3}$ Surgery, Hospital of the University of Pennsylvania; ${ }^{4}$ Perelman School of Medicine at the University of Pennsylvania, Philadelphia, Pennsylvania; and ${ }^{5}$ Department of Biochemistry, Purdue University, West Lafayette, Indiana

OBJECTIVE Intraoperative molecular imaging with tumor-targeted fluorescent dyes can enhance resection rates. In contrast to visible-light fluorophores (e.g., 5-aminolevulinic-acid), near-infrared (NIR) fluorophores have increased photon tissue penetration and less contamination from tissue autofluorescence. The second-window ICG (SWIG) technique relies on passive accumulation of indocyanine green (ICG) in neoplastic tissues. OTL38, conversely, targets folate receptor overexpression in nonfunctioning pituitary adenomas. In this study, we compare the properties of these 2 modalities for NIR imaging of pituitary adenomas to better understand the potential for NIR imaging in neurosurgery.

METHODS A total of 39 patients with pituitary adenomas were enrolled between June 2015 and January 2018 in 2, sequential, IRB-approved studies. Sixteen patients received systemic ICG infusions 24 hours prior to surgery, and another 23 patients received OTL38 infusions 2-3 hours prior to surgery. NIR fluorescence signal-to-background ratio (SBR) was recorded during and after resection. Immunohistochemistry was performed on the 23 adenomas resected from patients who received OTL38 to assess expression of folate receptor-alpha (FR $\alpha)$.

RESULTS All 16 adenomas operated on after ICG administration demonstrated strong NIR fluorescence (mean SBR $4.1 \pm 0.69$ [SD]). There was no statistically significant difference between the 9 functioning and 7 nonfunctioning adenomas $(p=0.9)$. After administration of OTL38, the mean SBR was $1.7 \pm 0.47$ for functioning adenomas, $2.6 \pm 0.91$ for all nonfunctioning adenomas, and $3.2 \pm 0.53$ for the subset of FR $\alpha$-overexpressing adenomas. Tissue identification with white light alone for all adenomas demonstrated $88 \%$ sensitivity and $90 \%$ specificity. SWIG demonstrated $100 \%$ sensitivity but only $29 \%$ specificity for both functioning and nonfunctioning adenomas. OTL38 was $75 \%$ sensitive and $100 \%$ specific for all nonfunctioning adenomas, but when assessment was limited to the 9 FR $\alpha$-overexpressing adenomas, the sensitivity and specificity of OTL38 were both $100 \%$.

CONCLUSIONS Intraoperative imaging with NIR fluorophores demonstrates highly sensitive detection of pituitary adenomas. OTL38, a folate-receptor-targeted fluorophore, is highly specific for nonfunctioning adenomas but has no utility in functioning adenomas. SWIG, which relies on passive diffusion into neoplastic tissue, is applicable to both functioning and nonfunctioning pituitary adenomas, but it is less specific than targeted fluorophores. Thus, targeted and nontargeted NIR fluorophores play important, yet distinct, roles in intraoperative imaging. Selectively and intelligently using either agent has the potential to greatly improve resection rates and outcomes for patients with intracranial tumors.

https://thejns.org/doi/abs/10.3171/2018.7.JNS181642

KEYWORDS folate receptor; indocyanine-green; near-infrared imaging; pituitary adenoma; targeted imaging; pituitary surgery

\footnotetext{
ABBREVIATIONS 5-ALA = 5-aminolevulinic acid; $\mathrm{ACTH}=$ adrenocorticotropic hormone; EPR = enhanced permeability and retention; FR $\alpha=$ folate receptor-alpha; GH = growth hormone; ICG = indocyanine green; NIR = near-infrared; NPV = negative predictive value; PPV = positive predictive value; SBR = signal-to-background ratio; SPECT = single-photon emission computed tomography; SWIG = second-window ICG; TSH = thyroid-stimulating hormone.
}

SUBMITTED June 6, 2018. ACCEPTED July 31, 2018.

INCLUDE WHEN CITING Published online December 14, 2018; DOI: 10.3171/2018.7.JNS181642. 
$\mathrm{N}$ EUROSURGICAL resection improves survival and/ or alleviates neurological symptoms for many patients with intracranial tumors. $9,10,13,18,20,33,35,37$ During these resections, distinguishing neoplastic from non-neoplastic tissue is a critical task of the neurosurgeon. Intraoperative molecular imaging using fluorescent dyes during oncological surgery may provide clinical benefit.

Use of 5-aminolevulinic acid (5-ALA), an FDA-approved visible-light-spectrum fluorescent dye, has been associated with enhanced resection rates and progressionfree survival in glioblastoma patients. ${ }^{26,38}$ Newer agents that fluoresce in the near-infrared (NIR) region have recently been studied for use in neurosurgery as well as in other surgical fields. Indocyanine green (ICG) is the only FDA-approved NIR fluorophore (peak emission $835 \mathrm{~nm}$ ) and has been widely used in vascular surgery for decades as an angiographic agent. Recently, our group has demonstrated that a high-dose injection of ICG 24 hours prior to surgery allows the normal tissues to clear ICG, while abnormal tumor tissue retains ICG; this allows real-time, intraoperative visualization of gliomas, meningiomas, and brain metastases with high sensitivity $(>85 \%) .{ }^{22-24} \mathrm{We}$ have termed this technique second-window ICG (SWIG).

A more recently developed NIR dye is OTL38 (On Target Laboratories), a modified folate analog linked to an ICG congener, which binds and is internalized by cells that overexpress folate receptors, such as ovarian carcinoma, lung adenocarcinoma, and nonfunctioning pituitary adenomas., ${ }^{7,11,25}$ Such receptor-specific fluorophores are currently under FDA investigational Phase I, II, and III studies. We have previously published an investigatorinitiated study on the use of OTL38 for patients with pituitary adenoma, which demonstrated high sensitivity and specificity. $^{21}$

In this study, we compare the signal characteristics of SWIG with OTL38 during pituitary adenoma surgery. We hypothesized that the NIR signal intensity would differ based on the dye administered, as the method of accumulation within tissues differs for the 2 agents. Furthermore, we hypothesized that both NIR agents could be used to determine the margin after resection with high sensitivity but that OTL38 would have utility only in nonfunctioning adenomas.

\section{Methods \\ Study Design}

Patients were enrolled in one of 2 sequential, separate clinical trials (June 2015-January 2017), one evaluating SWIG in intracranial tumors and the other a pilot study evaluating OTL38 administration in pituitary adenomas. Both studies are registered with clinical trials.gov; the registration number for the SWIG study is NCT03262636, and that for the OTL38 study is NCT02629549. Inclusion criteria were diagnosis of a pituitary adenoma and age $>18$ years. Pregnancy and history of allergy to folate (OTL38 study) were the main exclusion criteria. Between October 2015 and January 2017, all patients were enrolled in the OTL38 trial; otherwise, patients were enrolled in the SWIG trial. The 2 prospective cohort studies were each approved by the University of Pennsylvania institu- tional review board, and all patients gave informed consent. Patients underwent preoperative MRI of the brain/ sella with and without gadolinium contrast, as well as serum measurements of thyroid stimulating hormone (TSH), growth hormone (GH), adrenocorticotropic hormone (ACTH), and prolactin. Those with neither clinical signs of hormone oversecretion nor elevated serum levels were designated as having nonfunctioning adenomas. Patients were informed that enrollment in the study would not substantially change the extent of surgery.

\section{NIR Fluorophore Administration}

Patients were brought to the outpatient infusion suite prior to surgery-24 hours before surgery for the SWIG group and 2-3 hours before surgery for the OTL38 group. The patients in the OTL38 group were instructed to discontinue folate supplements or folate-containing multivitamins 48 hours prior to surgery in order to reduce potential interactions with OTL38. Patients were given 25 $\mathrm{mg}$ diphenhydramine intravenously as a precautionary measure and then, over the course of 1 hour, they received infusions of either $5 \mathrm{mg} / \mathrm{kg}$ of ICG or $0.025 \mathrm{mg} / \mathrm{kg}$ of OTL38. The patients' vital signs were monitored throughout the infusion and for 30 minutes afterward.

\section{NIR Imaging System}

In all cases, NIR signal was visualized using 2 different versions of the VisionSense Iridium camera system equipped with a 4-mm endoscope. This system is FDA approved for perfusion imaging in plastic and reconstructive surgery (to assess tissue flap vascularity, for example). Two different excitation laser sources were utilized. For OTL38, the excitation source is a laser tuned to $785 \mathrm{~nm}$ and the sensor is filtered for emission in the 800- to 835$\mathrm{nm}$ range. For ICG, the excitation source is a laser tuned to $805 \mathrm{~nm}$, and the sensor is filtered for emission in the same 820 - to $860-\mathrm{nm}$ range. The commercially available VisionSense system was recently compared to other NIR visualization systems, and its sensor gain and sensitivity are considered the best among the commercially available systems. ${ }^{4,5}$

\section{Surgical Approach and Study Procedure}

The endonasal endoscopic transsphenoid approach was performed by otorhinolaryngologists using standard endoscopic technique. ${ }^{6,32}$ Upon exposing the sella, the senior neurosurgeon (J.Y.K.L.) used the VisionSense endoscope to record NIR images. The dura was initially kept intact in order to visualize NIR signal through it. Once the dura was opened, NIR fluorescence within the tumor was visualized (Fig. 1). After tumor exposure, specimens were taken from the tumor mass and sent to pathology for histopathologic diagnosis. Surgery then proceeded in the standard-of-care manner without the use of NIR fluorescence.

After the tumor had been resected and the attending neurosurgeon was satisfied that a complete resection had been achieved based on white light visualization, NIR imaging was used to identify areas of residual fluorescence (Fig. 2). Areas were biopsied at the discretion of the senior surgeon, and specimens were coded (yes/no for NIR 

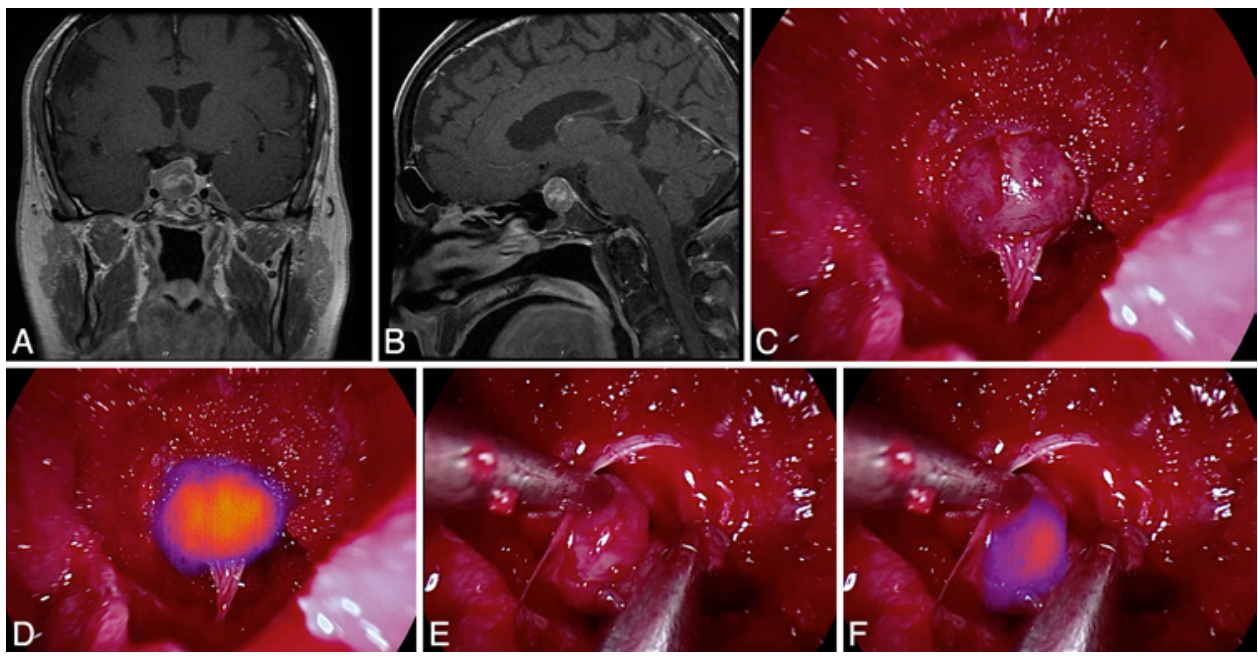

FIG. 1. Application of SWIG in a patient with a prolactin-staining nonfunctioning pituitary adenoma. A and B: Preoperative coronal (A) and sagittal (B) contrast-enhanced T1-weighted MR images demonstrating a 22-mm adenoma. C and D: Initial view of the tumor after opening the dura as visualized with white light (C) and with NIR imaging (D). NIR imaging delineates the tumor with a high SBR of 4.5. E and F: Margin specimen biopsied during surgery, viewed with white light (E) and with NIR imaging (F). Viewed with white light alone, the specimen did not convincingly appear neoplastic. With NIR imaging, however, the specimen fluoresced with an SBR of 3.2. The final pathological examination demonstrated that the specimen was neoplastic, suggesting that the NIR imaging was more sensitive for neoplasm in this case. Figure is available in color online only.

fluorescence and yes/no for tumor based on the surgeon's impression) and sent for pathological analysis. The closure was performed jointly by otorhinolaryngology and neurosurgery. Patients were admitted to the intensive care unit following surgery. A postoperative MRI study was performed on the first postoperative day and then after 2, 6, and/or 12 months.

\section{Immunohistochemistry for FR $\alpha$ in OTL38 Patients}

Tumor specimens from patients in the OTL38 arm were chemically fixed and embedded in paraffin blocks and sectioned at 5- $\mu \mathrm{m}$ thickness. Immunohistochemistry for FR $\alpha$ was performed using murine monoclonal antibodies against folate receptors (1:20 dilution of NCL-L-FRalpha; Leica Biosystems). In order to quantify FR $\alpha$ expression in each sample, H-scores were calculated by a single neuropathologist (M.N.). The H-score ranges from 0 through 300 , with 300 implying strong staining in all cells and 0 implying no staining in any cell..$^{2,29}$

\section{NIR Image Analysis for SBR}

The NIR imaging data were analyzed after the surgical procedure using the VisionSense software (VSPlayer v1.8.05.01; VisionSense) and ImageJ (https://imagej.nih. gov/ij/; National Institutes of Health) to calculate the signal-to-background ratio (SBR), as described in our previous paper. ${ }^{21}$ In brief, care was taken to visualize samples where the opticocarotid distance was $<50 \%$ of the entire screen. It was previously noted that false-positive detection of NIR fluorescence could be minimized by increasing the distance from the endoscope to the tissue. Thus, all initial images were taken with the medial opticocarotid recess spanning less than half of the screen, as our prior work demonstrated this to be the ideal distance..$^{15,17,21,41} \mathrm{Re}$ - gions of interest were drawn around the tumor and adjacent normal tissue (sometimes bone or mucosa).

\section{Data Analysis}

Statistical comparisons with linear regression, t-tests,
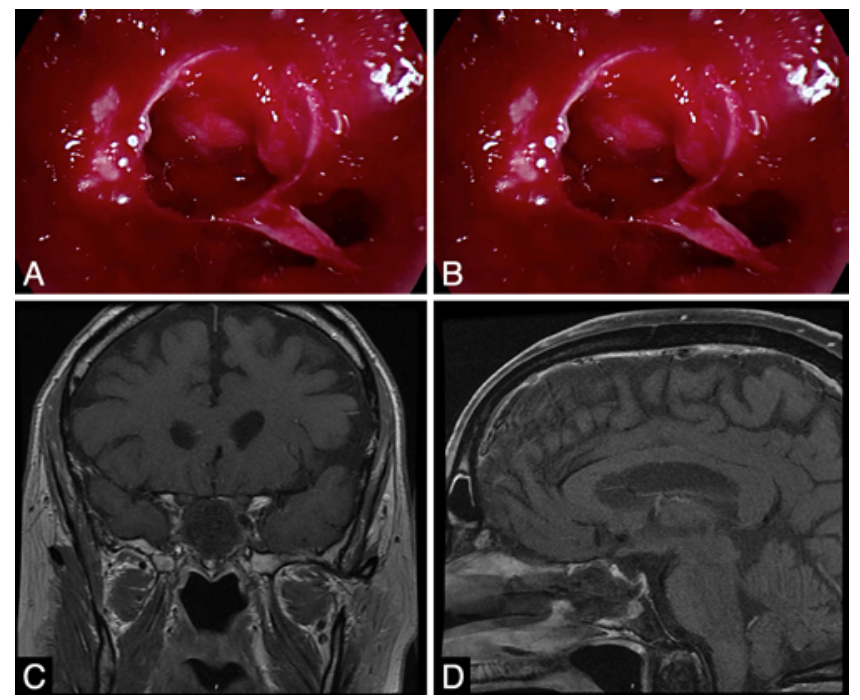

FIG. 2. Intraoperative and MR images obtained in the same patient as represented in Fig. 1 after resection of the pituitary adenoma. $A$ and B: Postresection view obtained with white light $(A)$ and $N I R(B)$ imaging prior to closure. The surgeon was satisfied that no residual tumor was present (A). NIR imaging demonstrated no residual areas of fluorescence (B). C and D: Postoperative day 1 coronal (C) and sagittal (D) contrast-enhanced T1-weighted MR images demonstrating gross-total resection. The absence of NIR fluorescence prior to closure correctly predicted absence of residual neoplasm. Figure is available in color online only. 
TABLE 1. Patient demographics and presenting symptoms in each study group

\begin{tabular}{lcc}
\hline \multicolumn{1}{c}{ Characteristic } & $\begin{array}{c}\text { OTL38 } \\
(23 \text { cases })\end{array}$ & $\begin{array}{c}\text { SWIG } \\
(16 \text { cases })\end{array}$ \\
\hline Male, $\%$ & 52 & 56 \\
\hline Mean age in yrs & $54.0 \pm 17.5$ & $57.4 \pm 11.0$ \\
\hline Mean adenoma size in mm & $24 \pm 13$ & $22 \pm 11$ \\
\hline Prior pituitary surgery, $\%$ & 17.4 & 18.8 \\
\hline Prior pituitary radiation, $\%$ & 0 & 0 \\
\hline $\begin{array}{l}\text { Mean time from contrast agent infusion } \\
\text { to visualization in hrs }\end{array}$ & $4.8 \pm 1.2$ & $22 \pm 2.4$ \\
\hline Nonfunctioning adenoma & 14 & 7 \\
\hline Mean adenoma size in mm & $27 \pm 13$ & $25 \pm 12$ \\
\hline Macroadenoma & 14 & 7 \\
\hline Visual field compromise & 6 & 2 \\
\hline Cavernous sinus invasion & 12 & 7 \\
\hline Functioning adenoma & 9 & 9 \\
\hline Mean adenoma size in mm & $18 \pm 11$ & $19 \pm 10$ \\
\hline Macroadenoma & 8 & 7 \\
\hline Cushing's disease & 6 & 2 \\
\hline Acromegaly & 3 & 4 \\
\hline Prolactinoma & 0 & 2 \\
\hline Thyrotroph adenoma & 0 & 1 \\
\hline
\end{tabular}

Data are numbers of cases unless otherwise indicated. Mean values are presented with SDs.

and nonparametric tests were performed using Stata 10 (StataCorp, LLC). Two-by-two contingency tables were constructed to calculate the sensitivity, specificity, positive predictive value (PPV), and negative predictive value (NPV) for the surgeon's impression, SWIG, and OTL38.

\section{Results}

Clinical Data

A total of 39 patients were enrolled between October 2015 and December 2017. Sixteen patients (7 with non- functioning, 4 with $\mathrm{GH}$-secreting, 2 with $\mathrm{ACTH}$-secreting, 2 with PRL-secreting adenomas, and 1 with a TSH-secreting adenoma) were enrolled in the SWIG trial and 23 patients (14 with nonfunctioning, 6 with ACTH-secreting, 3 with GH-secreting adenomas) were enrolled in the OTL38 trial (Table 1). SWIG was administered at a mean $( \pm$ SD) of $22 \pm 2.4$ hours before tumor visualization, and OTL38 was administered at a mean of $4.8 \pm 1.2$ hours before tumor visualization. All patients tolerated ICG or OTL38 infusions without adverse event and no permanent surgical complications were identified.

\section{Folate Receptor Immunohistochemistry}

After standard postoperative immunohistochemical staining for pituitary hormones, further immunohistochemistry for FR $\alpha$ was performed on paraffin-embedded tissue sections using the protocol described in Methods.

Non-neoplastic pituitary tissue from 11 specimens demonstrated a mean FR $\alpha \mathrm{H}$-score of $50 \pm 40$ (median $34)$. Nine of 23 adenomas in the OTL38 group demonstrated significant FR $\alpha$ overexpression (H-score $>150$ ), and all 9 were nonfunctioning adenomas. The other 14 adenomas (5 nonfunctioning, 6 ACTH-secreting, $3 \mathrm{GH}-$ secreting) demonstrated FR $\alpha$ H-scores $<100$.

\section{Intraoperative NIR Fluorescence}

The 16 adenomas in the patients who received ICG (SWIG trial) demonstrated NIR fluorescence through the dura that localized to the tumor with a mean SBR of 2.7 \pm 0.63 ; the SBR increased (to a mean value of $4.1 \pm 0.72$ ) upon dura opening and direct exposure of the tumor (Fig. 1). NIR wavelength is longer than visible light, and penetration of fluorescent signal through normal dura has been reported by our group. ${ }^{20-23}$ When the cases were grouped by preoperative hormonal status, the average SBR with tumor exposure was $4.1 \pm 0.63$ for the nonfunctioning adenomas and $4.0 \pm 0.82$ for the functioning adenomas $(p=0.9)$. A detailed breakdown of NIR SBR grouped by functional status is shown in Table 2. There were no significant differences in SWIG fluorescence signal between the classes of pituitary adenomas. Tumor specimens were further imaged ex vivo, using both gross specimen and

TABLE 2. Summary of NIR imaging with OTL38 and SWIG, stratified by adenoma type

\begin{tabular}{lccccc}
\hline \multirow{2}{*}{ Adenoma Type } & \multicolumn{2}{c}{ Dura View } & & \multicolumn{2}{c}{ Tumor View } \\
\cline { 2 - 3 } \cline { 5 - 6 } & OTL38 & SWIG & & OTL38 & SWIG \\
\hline Nonfunctioning adenoma & $1.9 \pm 0.39$ & $2.9 \pm 0.71$ & & $2.6 \pm 0.91$ & $4.1 \pm 0.63$ \\
\hline Functioning adenoma & $1.3 \pm 0.22$ & $2.5 \pm 0.5$ & & $1.7 \pm 0.47$ & $4.0 \pm 0.82$ \\
\hline Cushing's disease & $1.3 \pm 0.26$ & 2 & & $1.6 \pm 0.56$ & $4.0 \pm 0.84$ \\
\hline Acromegaly & $1.3 \pm 0.14$ & $2.7 \pm 0.55$ & & $1.9 \pm 0.15$ & $3.9 \pm 1.2$ \\
\hline Prolactinoma & $\mathrm{NA}$ & $\mathrm{NA}$ & & $\mathrm{NA}$ & $4.1 \pm 0.35$ \\
\hline Thyrotroph adenoma & $\mathrm{NA}$ & 2.3 & & $\mathrm{NA}$ & 4.6 \\
\hline Folate-receptor-overexpressing adenoma & $2.0 \pm 0.34$ & $\mathrm{NA}$ & & $3.2 \pm 0.53$ & $\mathrm{NA}$ \\
\hline Non-folate-receptor-overexpressing adenoma & $1.4 \pm 0.31$ & $\mathrm{NA}$ & & $1.6 \pm 0.42$ & $\mathrm{NA}$ \\
\hline Average SBR & $1.6 \pm 0.43$ & $2.7 \pm 0.63$ & & $2.2 \pm 0.88$ & $4.1 \pm 0.72$ \\
\hline
\end{tabular}

NA = not applicable.

Data presented as mean SBRs with SDs. 

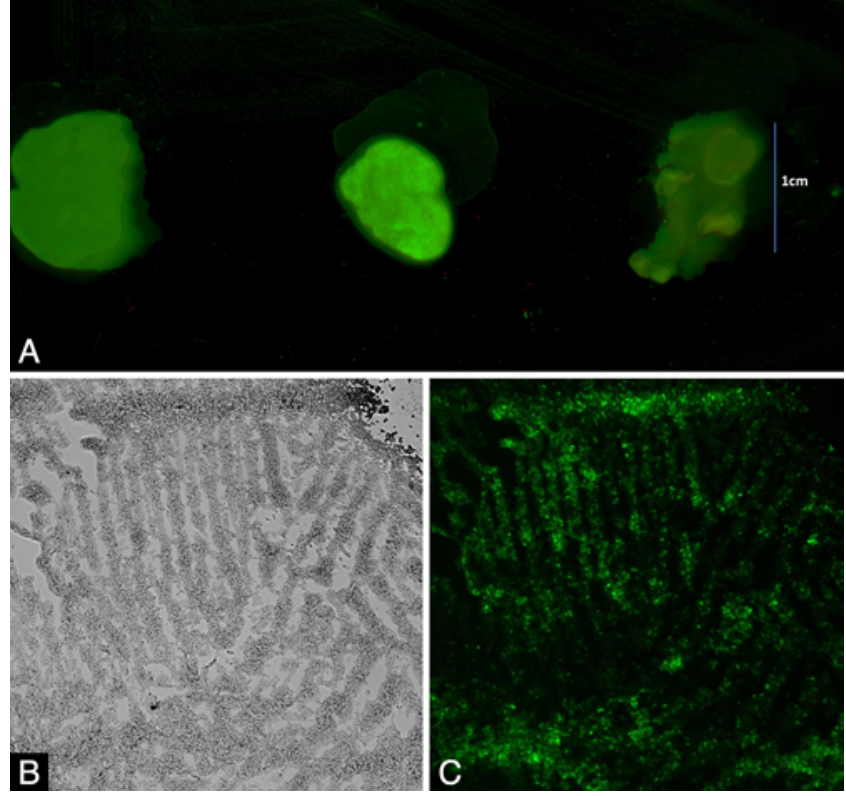

FIG. 3. Ex-vivo NIR imaging of pituitary adenoma specimens. A: Ex-vivo imaging of a specimen from a pituitary adenoma (middle) shows NIR fluorescence. A meningioma specimen (right) and a glioblastoma specimen (left) are shown for comparison. B and C: Microscopic imaging of the same pituitary adenoma specimen, under white light only $(B)$ and with NIR imaging $(C)$. Original magnification $\times 100$. Figure is available in color online only.

microscopic imaging (Fig. 3). Specimens that were fluorescent intraoperatively demonstrated strong fluorescence ex vivo. On microscopy, it was confirmed that areas of tumor demonstrated diffuse, rather than focal, uptake of ICG.

The 23 adenomas in the patients who received OTL38 (folate dye) fluoresced with a mean SBR of $1.6 \pm 0.43$ through the dura, and the average NIR SBR increased to
$2.2 \pm 0.88$ upon tumor exposure (Table 2). When the cases were grouped by preoperative hormonal status, the 14 nonfunctioning adenomas had a mean SBR of $2.6 \pm 0.91$, and the 9 functioning adenomas had a mean SBR of $1.7 \pm 0.47$ $(p=0.013)$. When the cases were grouped by postoperatively determined FR $\alpha$ expression, the 9 FR $\alpha$-overexpressing adenomas demonstrated a mean SBR of $3.2 \pm 0.53$, while the other 14 adenomas with low FR $\alpha$ expression had a mean SBR of $1.6 \pm 0.42(\mathrm{p}=0.0001)$ (Fig. 4).

\section{NIR Fluorescence Detection of Neoplastic Tissue}

A total of 30 specimens were sampled from the 16 patients enrolled in the SWIG trial. Each time, the specimens were labeled by the surgeon as tumor or not tumor based on visual and tactile assessment; the presence of NIR fluorescence in each specimen prior to biopsy was also recorded as yes or no (Table 3). Using postoperative histopathology as the gold-standard diagnosis of each specimen, the sensitivity, specificity, positive predictive value (PPV), and negative predictive value (NPV) were calculated. Using an NIR SBR cutoff of 2.0 (i.e., NIR signal in tissue is $>2 \times$ the background signal), SWIG was $100 \%$ sensitive $(95 \%$ CI $81 \%-100 \%)$ but only $29 \%$ specific $(95 \%$ CI $5.1 \%-70 \%)$ for neoplastic tissue, with a PPV of $82 \%$ (95\% CI 74\%-88\%) and an NPV of $100 \%$ (95\% CI 20\%-100\%). The preoperative hormonal status of the patient had no effect on intraoperative fluorescence. In nonfunctioning adenomas, SWIG was $100 \%$ sensitive but only $33 \%$ specific, with a PPV of $83 \%$ and NPV of $100 \%$; in functioning adenomas, SWIG was $100 \%$ sensitive and only $25 \%$ specific, with a PPV of $81 \%$ and NPV of $100 \%$.

A total of 48 specimens were sampled from the 23 patients enrolled in the OTL38 trial. Statistical analysis of NIR imaging using OTL38 was performed only on the 28 specimens from the 14 nonfunctioning adenomas, as OTL38 was hypothesized to have utility only in FR $\alpha$ overexpressing tumors, such as nonfunctioning adenomas.

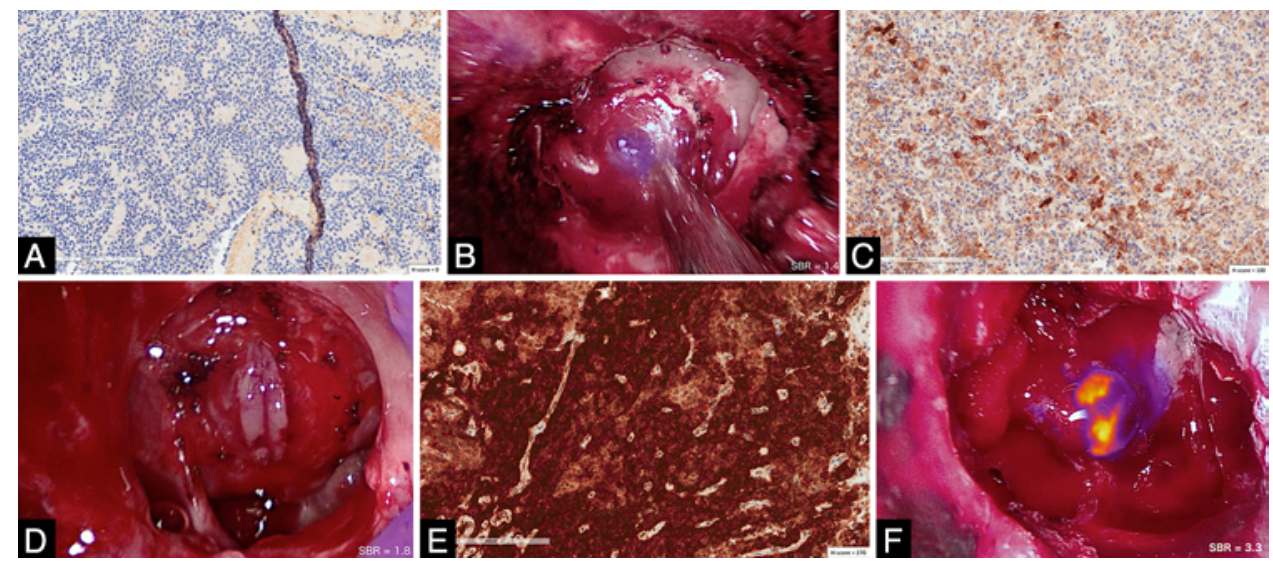

FIG. 4. Photomicrographs and intraoperative photographs showing correlation of $F R \alpha$ expression with OTL38 imaging signal in pituitary adenomas. A and B: This patient's adenoma stained for FR $\alpha$ with an $\mathrm{H}$-score of 0 , as no cells expressed any FR $\alpha(A)$. The SBR upon tumor exposure was 1.4 (B). C and D: This patient's adenoma stained for FR $\alpha$ with an $\mathrm{H}$-score of 100, indicating moderate FR $\alpha$ expression in some cells (C). The SBR upon tumor exposure was 1.8 (D). E and F: This patient's adenoma stained for $\mathrm{FR} \alpha$ with an $\mathrm{H}$-score of 270 , indicating high FR $\alpha$ expression in nearly all the cells (E). The SBR upon tumor exposure was 3.3 (F). Original magnification $\times 200$. Figure is available in color online only. 
TABLE 3. Sensitivity and specificity for neoplastic tissue using white-light-only visualization, NIR imaging with SWIG, and NIR imaging with OTL38

\begin{tabular}{|c|c|c|c|c|c|c|}
\hline \multirow[b]{2}{*}{ Modality \& Path Finding } & \multicolumn{2}{|c|}{$\begin{array}{c}\text { Tumor on Intraop } \\
\text { Visualization }\end{array}$} & \multicolumn{4}{|c|}{ Point Estimate $(95 \% \mathrm{Cl})$} \\
\hline & Yes & No & Sensitivity & Specificity & PPV & NPV \\
\hline \multicolumn{7}{|l|}{ White light, $n=78$} \\
\hline \multicolumn{7}{|l|}{ Tumor on path } \\
\hline Yes & 50 & 7 & \multirow{2}{*}{$88 \%(75-94 \%)$} & \multirow{2}{*}{$90 \%(68-98 \%)$} & \multirow{2}{*}{$96 \%(85-99 \%)$} & \multirow{2}{*}{$73 \%(51-88 \%)$} \\
\hline No & 2 & 19 & & & & \\
\hline \multicolumn{7}{|l|}{ SWIG fluorescence, $n=30$} \\
\hline \multicolumn{7}{|l|}{ Tumor on path } \\
\hline Yes & 23 & 0 & \multirow{2}{*}{$100 \%(81-100 \%)$} & \multirow{2}{*}{$29 \%(5.1-70 \%)$} & \multirow{2}{*}{$82 \%(74-88 \%)$} & \multirow{2}{*}{$100 \%(20-100 \%)$} \\
\hline No & 5 & 2 & & & & \\
\hline \multicolumn{7}{|c|}{$\begin{array}{l}\text { OTL38 fluorescence (nonfunctioning adenoma), } \\
n=28\end{array}$} \\
\hline \multicolumn{7}{|l|}{ Tumor on path } \\
\hline Yes & 15 & 5 & \multirow{2}{*}{$75 \%(51-90 \%)$} & \multirow{2}{*}{$100 \%(60-100 \%)$} & \multirow{2}{*}{$100 \%(75-100 \%)$} & \multirow{2}{*}{$62 \%(43-77 \%)$} \\
\hline No & 0 & 8 & & & & \\
\hline \multicolumn{7}{|c|}{$\begin{array}{l}\text { OTL38 fluorescence (FR } \alpha \text {-overexpressing } \\
\text { adenoma), } n=18\end{array}$} \\
\hline \multicolumn{7}{|l|}{ Tumor on path } \\
\hline Yes & 15 & 0 & \multirow{2}{*}{$100 \%$ (75-100\%) } & \multirow{2}{*}{$100 \%$ (31-100\%) } & \multirow{2}{*}{$100 \%(75-100 \%)$} & \multirow{2}{*}{$100 \%(31-100 \%)$} \\
\hline No & 0 & 3 & & & & \\
\hline
\end{tabular}

Path = pathology.

As above, specimens were coded for tissue identity and for presence of NIR fluorescence by the senior surgeon prior to biopsy. Using an NIR SBR cutoff of 2.0, OTL38 was $75 \%$ sensitive (95\% CI 51\%-90\%) and $100 \%$ specific (95\% CI 60\%-100\%) for neoplastic tissue, with a PPV of $100 \%(95 \%$ CI $75 \%-100 \%)$ and an NPV of $62 \%(95 \%$ CI $43 \%-77 \%)$.

When the analysis was further limited to the 18 specimens from the 9 FR $\alpha$-overexpressing adenomas, OTL38 was $100 \%$ sensitive (95\% CI $75 \%-100 \%)$ and $100 \%$ specific (95\% CI 31\%-100\%) for neoplastic tissue, with a PPV of $100 \%(95 \%$ CI $75 \%-100 \%)$ and an NPV of $100 \%(95 \%$ CI $31 \%-100 \%$ ).

Overall, 78 specimens from 39 patients were sampled. The senior surgeon's determination of tissue identity based on white light, visual, and tactile impression was $88 \%$ sensitive (95\% CI 75\%-94\%) and $90 \%$ specific $(95 \%$ CI 68\%-98\%) for neoplasm, with a PPV of $96 \%$ (95\% CI $85 \%-99 \%)$ and an NPV of $73 \%$ (95\% CI 51\%-88\%).

\section{NIR Imaging Prediction of Postoperative MRI}

Of the 16 patients in the SWIG group, 5 had no signs of residual neoplasm, 2 had questionable residual neoplasm, and 9 had signs of residual neoplasm on MR images obtained on postoperative day 1 as well as on follow-up imaging up to 12 months later. Post-resection NIR imaging data from 12 patients were analyzed, since for 4 of the patients, the NIR imaging system was unavailable for the final imaging after resection. Overall, NIR imaging demonstrated residual fluorescence for 8 of the 9 patients with definite residual neoplasm (1 false negative) and 2 of the 3 patients with no residual neoplasm (2 false positives). An example of a false positive is shown in Fig. 5, with a true negative in Fig. 2.

Of the 23 patients in the OTL38 group, 15 had no signs of residual neoplasm on postoperative MRI follow-up. Of the 9 patients with FR $\alpha$-overexpressing adenomas, 4 had no signs of residual neoplasm on postoperative MRI. In these 4 patients, no NIR fluorescence was detected in the surgical bed after resection, immediately prior to closure ( 0 false positive). In 4 of the 5 patients with residual neoplasm on postoperative MRI, NIR imaging immediately prior to closure revealed areas of fluorescence in the surgical bed in locations corresponding to the residual neoplasms (1 false negative). In adenomas without FR $\alpha$ overexpression, no significant fluorescence was detected regardless of operative outcome.

\section{Discussion}

Distinguishing neoplasm from benign parenchyma is an essential skill for neurosurgeons, as enhanced resection leads to superior patient outcomes. ${ }^{30}$ Traditionally, surgeons rely on tactile and visual sensations, enhanced by magnifying loupes or surgical microscopes, to achieve this discrimination. One common tool employed in almost all cases is neuronavigation, which uses preoperatively acquired MRI and helps the surgeons to plan the route of entry and to more precisely locate the tumor. However, grosstotal resection rates for pituitary tumors remain less than 

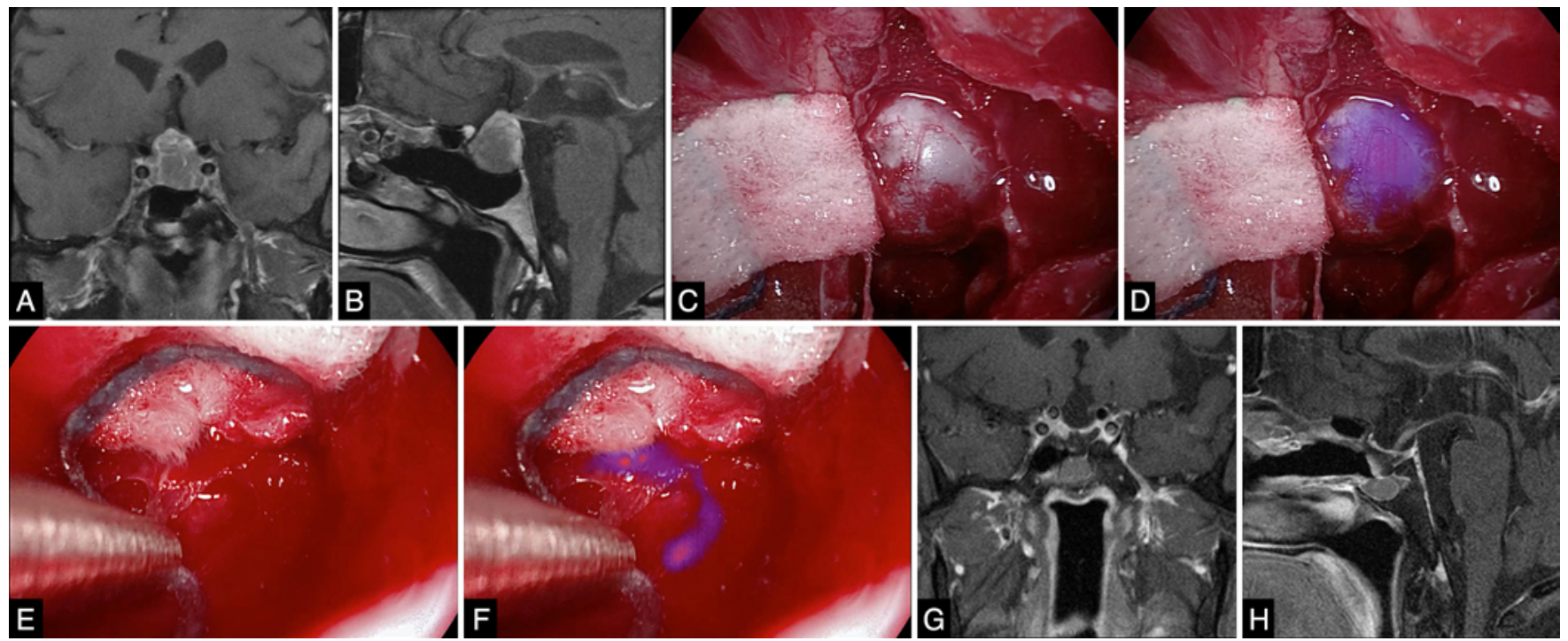

FIG. 5. SWIG in a patient with a null-cell nonfunctioning pituitary adenoma. A and B: Preoperative coronal (A) and sagittal (B) contrast-enhanced T1-weighted MR images demonstrating a 2-mm adenoma. C and D: Initial tumor view after opening the dura, with white light (C) and with NIR imaging (D). The NIR imaging delineates the tumor with a high SBR of 3.5 (D). E and F: Margin specimen biopsied during surgery, viewed with white light $(E)$ and with NIR imaging (F). With white light alone, the specimen did not appear neoplastic. With NIR imaging, the specimen fluoresced with an SBR of 2.5. The finding of final pathological examination was that the specimen was not neoplastic. Thus, the NIR imaging led to a false-positive signal in this case. G and H: Followup coronal $(G)$ and sagittal $(H)$ contrast-enhanced T1-weighted MR images obtained 3 months after surgery, demonstrating gross-total resection. Figure is available in color online only.

ideal (50\%-70\%), indicating that perhaps another technology is needed in the operating room. ${ }^{16}$ Intraoperative MRI is difficult and expensive to incorporate into the operating suite, and there is insufficient evidence supporting its use..$^{16}$ Here, we evaluate a novel technique, using NIR imaging and 2 different NIR fluorophores, in the transsphenoidal resection of pituitary adenomas.

\section{Rationale for NIR Fluorescent Molecular Imaging}

Intraoperative fluorescence imaging is an attractive option for visualizing neoplastic tissue, as it offers real-time information without significant additional cost or surgical time. NIR fluorophores have 2 main advantages over 5-ALA and other visible-light fluorophores: enhanced tissue penetration by the fluorescence ( $10 \mathrm{~mm}$ vs $\mu \mathrm{m}$ scale for 5-ALA) and lack of autofluorescence from surrounding benign parenchyma. ${ }^{39}$ Thus, NIR fluorescence imaging has the potential for improved SBR compared to visible light fluorophores; in addition, NIR fluorescence can be detected through normal dura, which is unlikely to occur with visible-light fluorophores. In this study, we demonstrate high SBR > 3-4, even when using a 4-mm endoscope. The physical constraints of a 4-mm endoscope have not been discussed in this analysis, but suffice it to say that excitation light power and the capture of photon energy on NIR sensors is less optimal with constrained 4-mm endoscopes compared to $10-\mathrm{mm}$ laparoscopes/thoracoscopes or exoscopes. ${ }^{31}$

\section{SWIG Is Highly Sensitive for Neoplasm}

Indocyanine-green (ICG) is an FDA-approved NIR dye (peak emission $835 \mathrm{~nm}$ ) that has been used for decades in vascular surgery for real-time vasculature imaging. When infused at a higher dose 24 hours prior to surgery, ICG accumulates in neoplastic tissue. Our group has demonstrated that SWIG can be used for various intracranial tumors, including metastases, glioblastomas, and meningiomas. It is hypothesized that SWIG works through the enhanced permeability and retention (EPR) effect, which stipulates that enhanced vasculature permeability allows normally impermeable particles to diffuse into and be retained by tumor tissue, whereas normal tissue with less permeable vasculature will show decreased retention of these more macroscopic materials (Fig. 6A). ${ }^{10,27,28}$ Thus, the very high dose of ICG administered 24 hours prior to surgery allows microaggregates of ICG to diffuse into and be retained within tumors with leaky vasculature, permitting real-time intraoperative visualization of the fluorescence. Our group previously demonstrated that there is a strong correlation between gadolinium enhancement of tumors on MRI and intraoperative SWIG fluorescence intensity, further supporting the EPR effect's role in SWIG. ${ }^{24}$ Since pituitary adenomas are considered hypo-enhancing relative to the normal pituitary gland, our initial hypothesis for this study was that SWIG would not provide sufficient ICG accumulation for visualization. ${ }^{34}$ We were thus pleasantly surprised to find in this study that ICG did accumulate with high sensitivity. We speculate that pituitary adenoma tissue is sufficiently abnormal in its clearance mechanisms that ICG accrues over 24 hours.

In our previous publications, we showed that SWIG is highly sensitive for intracranial neoplastic tissue, with a sensitivity $>85 \%$ for detection of the gross tumor and margin specimens. ${ }^{22-24}$ However, SWIG has low specificity 


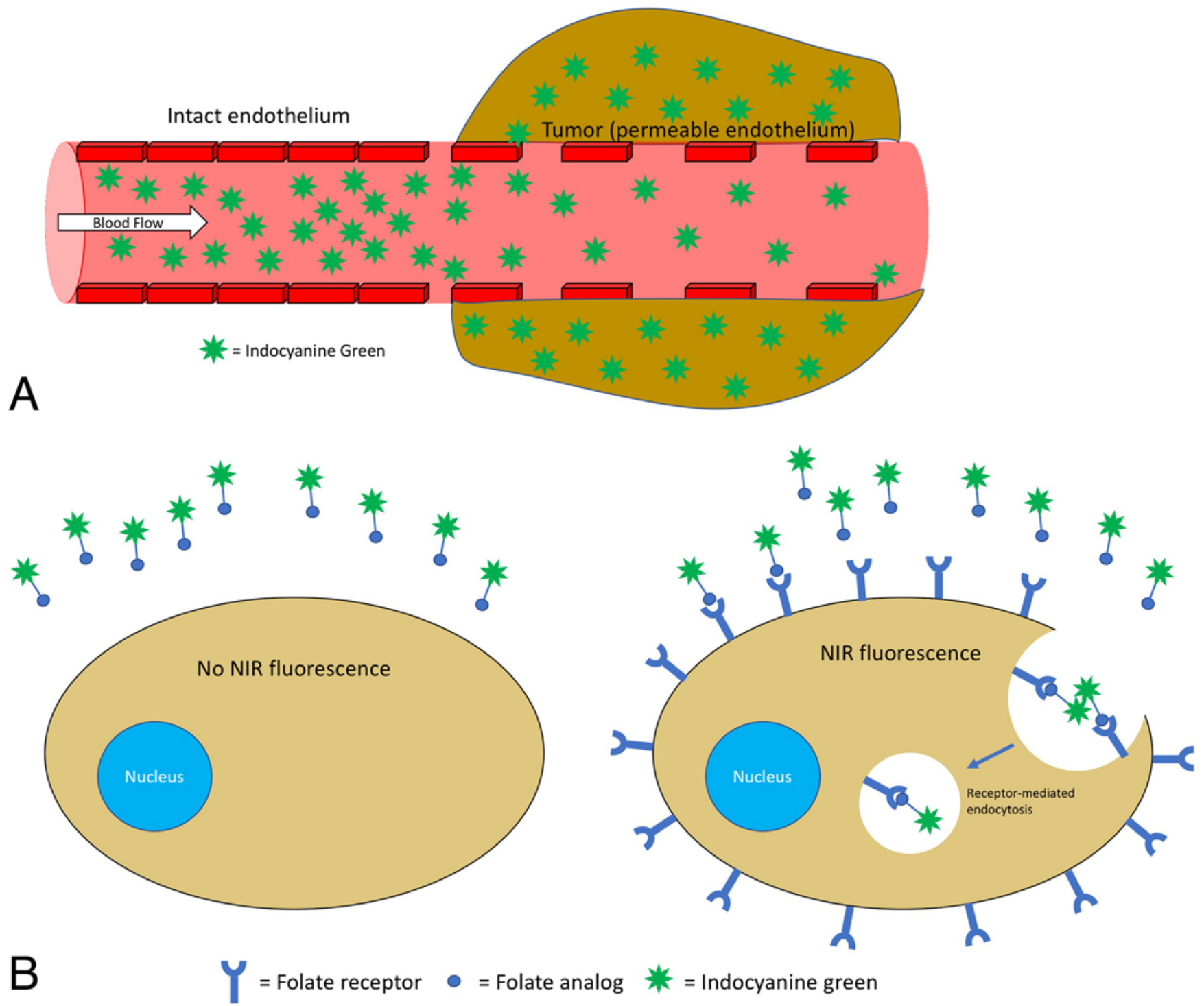

FIG. 6. Passive versus receptor-targeted delivery of NIR agent. A: ICG is hypothesized to be delivered to tumor tissues via the EPR effect. Benign tissue with intact vasculature and endothelium are minimally permeable to the NIR dye, but tumor tissue with disorganized and/or damaged vasculature allows the NIR dye to diffuse into the tissue and be retained. Thus, delayed intraoperative imaging 24 hours after systemic injection of ICG allows visualization of tumor tissue with permeable vasculature. B: OTL38 is a folate analog linked to ICG. Thus, it binds to and is internalized by cells that overexpress folate receptors but does not bind to cells that do not overexpress folate receptors. This allows OTL38 to specifically accumulate within tumor tissue with folate-receptor overexpression, such as nonfunctioning pituitary adenoma cells. Figure is available in color online only.

(25\%-38.9\% in our prior studies), due to a high incidence of false-positive signals. Similarly, in this study, SWIG had $100 \%$ sensitivity, $29 \%$ specificity, $82 \%$ PPV, and $100 \%$ NPV for pituitary adenomas. The surgeon's impression, for comparison, was $88 \%$ sensitive and $90 \%$ specific, with 96\% PPV and 73\% NPV. Similarly, in the final NIR imaging prior to surgical closure, SWIG demonstrated a falsepositive signal in 2 of 3 patients with no residual tumor on postoperative MRI. The low specificity and PPV of SWIG is thus interpreted cautiously, with the recognition that enhanced vasculature permeability is not a specific finding in tumors; inflammation and tissue injury can also lead to increased permeability, potentially causing false-positive signals. ${ }^{14}$ Indeed, in unpublished data, the senior surgeon has also recognized that while normal pituitary gland may not demonstrate significant ICG accumulation, the pituitary stalk and pars nervosa does often demonstrate nonspecific ICG accumulation. Biopsy of these areas has been limited by the unwillingness of the senior surgeon to harvest potentially normal and highly functional neural tissue.

SWIG's low specificity and PPV have implications regarding its utility. In many surgical oncology subspecialties, surrounding benign tissue is often sacrificed during resection of tumors, as the benefits of maximal resection usually outweigh the disadvantages of the iatrogenic damage. In neurosurgery, however, supramarginal resection is not considered standard-of-care, and thus, a highly sen- 
TABLE 4. Comparison of important characteristics of SWIG and OTL38 in clinical use

\begin{tabular}{lll}
\hline \multicolumn{1}{c}{ Characteristic } & \multicolumn{1}{c}{ SWIG } & OTL38 \\
\hline $\begin{array}{c}\text { Mechanism of delivery } \\
\text { to tumor cells }\end{array}$ & $\begin{array}{c}\text { Passive delivery via enhanced-permeability-and-retention } \\
\text { effect }\end{array}$ & $\begin{array}{c}\text { Active uptake by cells w/ folate-receptor overexpression, } \\
\text { due to the folate-analog moiety }\end{array}$ \\
\hline Average SBR & $4.1 \pm 0.69$ & $3.2 \pm 0.52$ (in FR $\alpha$-overexpressing adenoma) \\
\hline Sensitivity/NPV & $100 \% / 100 \%$ & $75 \% / 62 \%(100 \% / 100 \%$ in FR $\alpha$-overexpressing adenoma) \\
\hline Specificity/PPV & $29 \% / 82 \%$ & $100 \% / 100 \%$ \\
\hline Clinical applicability & $\begin{array}{c}\text { Widely applicable to intracranial tumors (metastases, } \\
\text { meningiomas, gliomas, functioning pituitary adenomas, } \\
\text { nonfunctioning pituitary adenomas) }\end{array}$ & $\begin{array}{c}\text { Limited in utility to tumors that overexpress folate receptors } \\
\text { (nonfunctioning pituitary adenomas, meningiomas) }\end{array}$ \\
\hline
\end{tabular}

sitive and specific method of tumor identification would benefit neurosurgeons. We do not recommend chasing after all areas of NIR fluorescence due to SWIG's lack of specificity. We believe, however, that SWIG does have the potential to complement the surgeon's armamentarium due to its high NPV. In our study, the NPV for the surgeon with white light alone was $73 \%$, indicating that in some cases benign tissue was considered to be neoplastic and resected. SWIG, on the other hand, demonstrated $100 \%$ NPV; thus, areas that do not fluoresce with SWIG are likely to be benign tissue and should not be resected. By combining the surgeon's high PPV and SWIG's high NPV for distinguishing neoplastic from benign tissue, neurosurgeons could avoid resection of normal tissue while still aiming for gross-total resection. Furthermore, we have demonstrated here that SWIG is widely applicable to all pituitary adenomas, as the preoperative hormonal status of the patient did not affect the NIR signal or the sensitivity, specificity, PPV, or NPV of intraoperative imaging with SWIG.

Hence, we recommend performing surgery based on white light visualization, and using SWIG for confirmation of degree of resection. If there is no residual NIR fluorescence, the surgeon can be very confident that resection has been thorough. In contrast, however, if there is residual NIR fluorescence, the surgeon must interpret this finding with extreme caution, recognizing the lack of specificity of SWIG.

\section{Folate-Receptor-Targeted Imaging Is Highly Sensitive and Specific for Neoplasm}

OTL38 is a folate analog linked to an ICG analog that binds and is internalized by cells that overexpress folate receptors (Fig. 6B) ${ }^{19,42}$ Folate is a crucial vitamin for cellular growth, replication, and turnover. Folate receptors exist in 4 forms (FR $\alpha, F R \beta, F R \gamma$, and FR $\delta$ ), and FR $\alpha$ has been reported to be overexpressed in ovarian, lung, and breast carcinomas. ${ }^{1,19,36,40}$ In a series of pioneering studies, it was further shown that nonfunctioning pituitary adenomas overexpress FR $\alpha^{7,8}$ Folate receptors have been successfully targeted for molecular imaging in single-photon emission computed tomography (SPECT), suggesting that they are valid cellular targets. ${ }^{11}$ Thus, we decided to attempt folate-receptor-targeted NIR imaging in pituitary adenomas, using OTL38.

In our group of 14 nonfunctioning adenomas, OTL38 was less sensitive than SWIG (75\%) for detecting neo- plasm, but more specific (100\%), with a PPV of $100 \%$ and an NPV of $62 \%$. The major reason for the reduced sensitivity and NPV was that 5 of the 14 nonfunctioning adenomas in our study did not overexpress FR $\alpha$ on postoperative immunohistochemistry. Since OTL38 can only bind cells that overexpress FR $\alpha$, NIR signal was predictably unreliable in those 5 cases. When analysis was limited to the 9 FR $\alpha$-overexpressing adenomas, the sensitivity, specificity, PPV, and NPV were all 100\% with OTL38.

Overall, OTL38 imaging demonstrated a superior ability for tissue identification compared to both the surgeon's impression alone and SWIG, with higher specificity and PPV. This makes intuitive sense, as OTL38 was designed to specifically bind $\mathrm{FR} \alpha$ rather than rely on the nonspecific EPR effect, like SWIG. While the NIR signal-tobackground ratio (SBR) is lower with OTL38 than with SWIG $(3.2 \pm 0.52$ vs $4.1 \pm 0.72, p=0.003)$, this difference may not be clinically significant, as our experience shows that any tumor with SBR > 2.5 can easily be visualized. Table 4 summarizes the important comparisons between SWIG and OTL38.

However, precisely because OTL38 specifically binds FR $\alpha$, its application is limited to certain tumor types. We have previously demonstrated the utility of SWIG in metastases, meningiomas, and gliomas, and now in pituitary adenomas, both functioning and nonfunctioning. ${ }^{22-24}$ Conversely, OTL38 imaging, as explained above, was effective in only 9 of the 23 patients who received OTL38. As suggested by Evans et al. and confirmed in our previous study, only nonfunctioning pituitary adenomas overexpress FR $\alpha$ (64\% in our series). ${ }^{7,8,21}$ While FR $\alpha$-targeted tomographic imaging with SPECT is capable of estimating FR $\alpha$ expression levels preoperatively, the cost-to-benefit analysis of preoperatively scanning all patients is unclear. ${ }^{11}$ Instead, we have data ${ }^{3}$ suggesting that initial NIR SBR of $>2.5$ upon tumor exposure may predict FR $\alpha$ overexpression with near- $100 \%$ probability; thus, a reasonable and affordable approach to using OTL38 clinically might be to administer it to all patients with nonfunctioning adenomas and determine intraoperatively whether each adenoma overexpresses FR $\alpha$ to decide if OTL38 fluorescence would be reliable for that surgery. If the initial SBR prior to resection is $<2.5$, resection should proceed using conventional techniques without NIR imaging.

\section{Limitations}

There are currently 2 major limitations to intraopera- 
tive NIR imaging. First, the fluorescent tissue must be in the line of sight of the endoscope in order to be excited and visualized. With the VisionSense Iridium system currently lacking angled endoscopes, we have discovered that it is difficult to visualize residual tumor around the cavernous sinuses. We believe this explains the one case in which OTL38 fluorescence failed to predict the resection outcome in a FR $\alpha$-overexpressing adenoma, as that patient's residual tumor abutted the cavernous sinus. We expect that the addition of an angled endoscope will address this limitation. In craniotomies using exoscopes, this is not an issue.

A second limitation is the low specificity of NIR imaging using SWIG. While this is expected due to its mechanism, the reduced specificity limits SWIG's reliability. Potential solutions are discussed below.

\section{Future Direction}

Advancement in NIR imaging requires the development of both novel fluorophores and advanced imaging modalities. While nonspecific dyes like ICG are widely applicable and have the potential to complement the surgeon's eyes and hands during surgery, appropriate molecular targets for targeted imaging should also be developed, as targeted imaging is much more specific and could help reduce iatrogenic neurological damage.

Furthermore, our group and collaborators are currently working to develop a tomographic NIR imaging system using spatial frequency domain tomography. Such tomographic imaging, like MRI, would provide depth information and allow the surgeon to assess the thickness of any fluorescent areas. We believe this would be useful in reducing false-positive signals from NIR fluorescence by allowing surgeons to distinguish true residual neoplasm from superficial spillage of fluorophores.

\section{Conclusions}

Intraoperative imaging with NIR fluorophores, targeted and nontargeted, demonstrates highly sensitive detection of pituitary adenomas. Second-window ICG (systemic infusion of ICG), which relies on passive diffusion into neoplastic tissue, is applicable to many tumor types but has a low specificity for detecting neoplasm. OTL38, a folatereceptor-targeted NIR fluorophore, is highly sensitive and specific for FR $\alpha$-overexpressing nonfunctioning adenoma tissue but has limited utility in functioning adenomas. Thus, targeted and nontargeted NIR fluorophores play important, yet distinct, roles in intraoperative imaging.

\section{Acknowledgments}

Supported in part by the National Institutes of Health R01 CA193556 (SS), and the Institute for Translational Medicine and Therapeutics of the Perelman School of Medicine at the University of Pennsylvania (JYKL). In addition, research reported in this publication was supported by the National Center for Advancing Translational Sciences of the National Institutes of Health under Award Number UL1TR000003 (JKYL). The content is solely the responsibility of the authors and does not necessarily represent the official views of the NIH.

\section{References}

1. Boogerd LSF, Boonstra MC, Beck AJ, Charehbili A, Hoogstins CES, Prevoo HAJM, et al: Concordance of folate receptor- $\alpha$ expression between biopsy, primary tumor and metastasis in breast cancer and lung cancer patients. Oncotarget 7:17442-17454, 2016

2. Budwit-Novotny DA, McCarty KS, Cox EB, Soper JT, Mutch DG, Creasman WT, et al: Immunohistochemical analyses of estrogen receptor in endometrial adenocarcinoma using a monoclonal antibody. Cancer Res 46:5419-5425, 1986

3. Cho SS, Zeh R, Pierce JT, Jeon J, Nasrallah M, Adappa ND, et al: Folate receptor near-infrared optical imaging provides sensitive and specific intraoperative visualization of nonfunctional pituitary adenomas. Oper Neurosurg (Hagerstown) [epub ahead of print], 2018

4. Cho SS, Zeh R, Pierce JT, Salinas R, Singhal S, Lee JYK: Comparison of near-infrared imaging camera systems for intracranial tumor detection. Mol Imaging Biol 20:213-220, 2018

5. DSouza AV, Lin H, Henderson ER, Samkoe KS, Pogue BW: Review of fluorescence guided surgery systems: identification of key performance capabilities beyond indocyanine green imaging. J Biomed Opt 21:80901, 2016

6. Elhadi AM, Hardesty DA, Zaidi HA, Kalani MYS, Nakaji P, White WL, et al: Evaluation of surgical freedom for microscopic and endoscopic transsphenoidal approaches to the sella. Neurosurgery 11 (Suppl 2):69-79, 2015

7. Evans CO, Reddy P, Brat DJ, O'Neill EB, Craige B, Stevens VL, et al: Differential expression of folate receptor in pituitary adenomas. Cancer Res 63:4218-4224, 2003

8. Evans CO, Yao C, Laborde D, Oyesiku NM: Folate receptor expression in pituitary adenomas: cellular and molecular analysis. Vitam Horm 79:235-266, 2008

9. Ezzat S, Asa SL, Couldwell WT, Barr CE, Dodge WE, Vance ML, et al: The prevalence of pituitary adenomas: a systematic review. Cancer 101:613-619, 2004

10. Fang J, Nakamura H, Maeda H: The EPR effect: unique features of tumor blood vessels for drug delivery, factors involved, and limitations and augmentation of the effect. Adv Drug Deliv Rev 63:136-151, 2011

11. Galt JR, Halkar RK, Evans CO, Osman NA, LaBorde D, Fox $\mathrm{TH}$, et al: In vivo assay of folate receptors in nonfunctional pituitary adenomas with ${ }^{99 \mathrm{~m} T c-f o l a t e ~ S P E C T / C T . ~ J ~ N u c l ~ M e d ~}$ 51:1716-1723, 2010

12. Greenman Y, Cooper O, Yaish I, Robenshtok E, Sagiv N, Jonas-Kimchi T, et al: Treatment of clinically nonfunctioning pituitary adenomas with dopamine agonists. Eur J Endocrinol 175:63-72, 2016

13. Hammouche S, Clark S, Wong AHL, Eldridge P, Farah JO: Long-term survival analysis of atypical meningiomas: survival rates, prognostic factors, operative and radiotherapy treatment. Acta Neurochir (Wien) 156:1475-1481, 2014

14. Holt D, Okusanya O, Judy R, Venegas O, Jiang J, DeJesus E, et al: Intraoperative near-infrared imaging can distinguish cancer from normal tissue but not inflammation. PLoS One 9:e103342, 2014

15. Kikuchi R, Toda M, Wakahara S, Fujiwara H, Jinzaki M, Yoshida K: Analysis of the medial opticocarotid recess in patients with pituitary macroadenoma using three-dimensional images. World Neurosurg 93:139-143, 2016

16. Kuo JS, Barkhoudarian G, Farrell CJ, Bodach ME, Tumialan LM, Oyesiku NM, et al: Congress of Neurological Surgeons systematic review and evidence-based guideline on surgical techniques and technologies for the management of patients with nonfunctioning pituitary adenomas. Neurosurgery 79:E536-E538, 2016

17. Labib MA, Prevedello DM, Fernandez-Miranda JC, Sivakanthan S, Benet A, Morera V, et al: The medial opticocarotid recess: an anatomic study of an endoscopic "key landmark" 
for the ventral cranial base. Neurosurgery 72 (1 Suppl Operative):66-76, 2013

18. Lacroix M, Abi-Said D, Fourney DR, Gokaslan ZL, Shi W, DeMonte F, et al: A multivariate analysis of 416 patients with glioblastoma multiforme: prognosis, extent of resection, and survival. J Neurosurg 95:190-198, 2001

19. Ledermann JA, Canevari S, Thigpen T: Targeting the folate receptor: diagnostic and therapeutic approaches to personalize cancer treatments. Ann Oncol 26:2034-2043, 2015

20. Lee JYK, Bohman LE, Bergsneider M: Contemporary neurosurgical techniques for pituitary tumor resection. J Neurooncol 117:437-444, 2014

21. Lee JYK, Cho SS, Zeh R, Pierce JT, Martinez-Lage M, Adappa ND, et al: Folate receptor overexpression can be visualized in real time during pituitary adenoma endoscopic transsphenoidal surgery with near-infrared imaging. J Neurosurg 129:390-403, 2018

22. Lee JYK, Pierce JT, Thawani JP, Zeh R, Nie S, MartinezLage M, et al: Near-infrared fluorescent image-guided surgery for intracranial meningioma. J Neurosurg 128:380390, 2018

23. Lee JYK, Pierce JT, Zeh R, Cho SS, Salinas R, Nie S, et al: Intraoperative near-infrared optical contrast can localize brain metastases. World Neurosurg 106:120-130, 2017

24. Lee JYK, Thawani JP, Pierce J, Zeh R, Martinez-Lage M, Chanin M, et al: Intraoperative near-infrared optical imaging can localize gadolinium-enhancing gliomas during surgery. Neurosurgery 79:856-871, 2016

25. Liu X, Ma S, Yao Y, Li G, Feng M, Deng K, et al: Differential expression of folate receptor alpha in pituitary adenomas and its relationship to tumor behavior. Neurosurgery 70:1274-1280, 2012

26. Madajewski B, Judy BF, Mouchli A, Kapoor V, Holt D, Wang $\mathrm{MD}$, et al: Intraoperative near-infrared imaging of surgical wounds after tumor resections can detect residual disease. Clin Cancer Res 18:5741-5751, 2012

27. Maeda H: Enhanced permeability and retention (EPR) effect: basis for drug targeting to tumors, in Muzykantov VR, Torchilin VP (eds): Biomedical Aspects of Drug Targeting. New York: Springer, 2002, pp 211-228

28. Maeda H, Sawa T, Konno T: Mechanism of tumor-targeted delivery of macromolecular drugs, including the EPR effect in solid tumor and clinical overview of the prototype polymeric drug SMANCS. J Control Release 74:47-61, 2001

29. McCarty KS Jr, Szabo E, Flowers JL, Cox EB, Leight GS, Miller L, et al: Use of a monoclonal anti-estrogen receptor antibody in the immunohistochemical evaluation of human tumors. Cancer Res 46 (8 Suppl):4244s-4248s, 1986

30. Mirimanoff RO, Dosoretz DE, Linggood RM, Ojemann RG, Martuza RL: Meningioma: analysis of recurrence and progression following neurosurgical resection. J Neurosurg 62:18-24, 1985

31. Nishiyama K: From exoscope into the next generation. J Korean Neurosurg Soc 60:289-293, 2017

32. O'Malley BW Jr, Grady MS, Gabel BC, Cohen MA, Heuer GG, Pisapia J, et al: Comparison of endoscopic and microscopic removal of pituitary adenomas: single-surgeon experience and the learning curve. Neurosurg Focus 25(6):E10, 2008
33. Patchell RA: The management of brain metastases. Cancer Treat Rev 29:533-540, 2003

34. Peck WW, Dillon WP, Norman D, Newton TH, Wilson CB: High-resolution MR imaging of pituitary microadenomas at 1.5 T: experience with Cushing disease. AJR Am J Roentgenol 152:145-151, 1989

35. Sanai N, Polley MY, McDermott MW, Parsa AT, Berger MS: An extent of resection threshold for newly diagnosed glioblastomas. J Neurosurg 115:3-8, 2011

36. Shi H, Guo J, Li C, Wang Z: A current review of folate receptor alpha as a potential tumor target in non-small-cell lung cancer. Drug Des Devel Ther 9:4989-4996, 2015

37. Simpson D: The recurrence of intracranial meningiomas after surgical treatment. J Neurol Neurosurg Psychiatry 20:22-39, 1957

38. Stummer W, Pichlmeier U, Meinel T, Wiestler OD, Zanella F, Reulen HJ: Fluorescence-guided surgery with 5-aminolevulinic acid for resection of malignant glioma: a randomised controlled multicentre phase III trial. Lancet Oncol 7:392401,2006

39. Stummer W, Suero Molina E: Fluorescence imaging/agents in tumor resection. Neurosurg Clin N Am 28:569-583, 2017

40. Toffoli G, Cernigoi C, Russo A, Gallo A, Bagnoli M, Boiocchi M: Overexpression of folate binding protein in ovarian cancers. Int J Cancer 74:193-198, 1997

41. Yang Y, Zhan G, Liao J, Dang R, Wang H, Li Y, et al: Morphological characteristics of the sphenoid sinus and endoscopic localization of the cavernous sinus. J Craniofac Surg 26:1983-1987, 2015

42. Zhao R, Matherly LH, Goldman ID: Membrane transporters and folate homeostasis: intestinal absorption and transport into systemic compartments and tissues. Expert Rev Mol Med 11:e4, 2009

\section{Disclosures}

Dr. Lee reports owning stock options in VisionSense. Dr. Low reports direct stock ownership in OnTarget Laboratories.

\section{Author Contributions}

Conception and design: Lee, Singhal. Acquisition of data: Lee, Cho, Jeon, Buch, Nag, Nasrallah. Analysis and interpretation of data: Cho, Jeon. Drafting the article: Cho. Critically revising the article: Lee, Low. Approved the final version of the manuscript on behalf of all authors: Lee. Statistical analysis: Cho. Administrative/technical/material support: Low, Grady. Study supervision: Lee, Grady.

\section{Supplemental Information}

Previous Presentations

Portions of this work were presented in abstract form at the AANS 2018 meeting in New Orleans, Louisiana, on May 2, 2018.

\section{Correspondence}

John Y. K. Lee: Hospital of the University of Pennsylvania, Philadelphia,PA.leejohn@uphs.upenn.edu. 